Research Article

\title{
Bioinformatics Analysis to Screen the Key Prognostic Genes in Tumor Microenvironment of Bladder Cancer
}

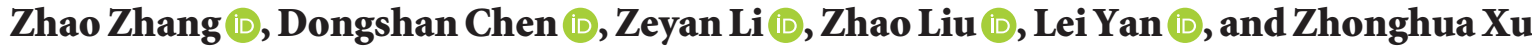 \\ Department of Urology, Qilu Hospital of Shandong University, Wenhuaxi Road 107\#, Jinan 250012, China \\ Correspondence should be addressed to Lei Yan; yanlei5309@126.com and Zhonghua Xu; xuzhonghua1963@163.com
}

Zhao Zhang and Dongshan Chen contributed equally to this work.

Received 26 October 2019; Accepted 16 January 2020; Published 17 February 2020

Academic Editor: Pradeep Tyagi

Copyright ( 2020 Zhao Zhang et al. This is an open access article distributed under the Creative Commons Attribution License, which permits unrestricted use, distribution, and reproduction in any medium, provided the original work is properly cited.

Bladder cancer (BLCA) is the fifth most common cancer and has the features of low survival rate and high morbidity and mortality. The Cancer Genome Atlas (TCGA) is a pool of global gene expression profile and contains huge amounts of cancer genomics data, which makes it possible to inquire the relationship between gene expression and prognosis of a series of malignant tumors including BLCA. Immune and stromal cells are two major components of tumor microenvironment (TME) which play an important role in judging the prognosis of tumor and influencing the progression of malignant, inflammatory, and metabolic disorders. In our study, we conducted a quantitative analysis of immune and stromal elements based on the ESTIMATE algorithm and thus divided BLCA cases into high and low groups. Then the differentially expressed genes closely related to tumor prognosis between groups were identified and had been shown to correlate with immune response and stromal alterations, which was further confirmed by functional enrichment analysis and protein-protein interaction networks. We validated those genes through BLCA dates downloaded from ArrayExpress and thus got the marker genes to predict prognosis of BLCA. Additionally, immune cell infiltration analysis explored the correlation between the verified genes and immune cells. In conclusion, we identified a series of TME-related genes that assess the prognosis and explored the interaction between TME and tumor prognosis to guide clinical individualized treatment.

\section{Introduction}

Bladder cancer is the most common malignancy of the urinary tract, and the diagnostics, treatment, and five-year survival rates for bladder cancer are largely unchanged since the 1990s [1]. Approximately $50 \%$ of those patients will have a recurrence in 2 years after an initial diagnosis and $16-25 \%$ will relapse after transurethral resection [2]. Although its exact mechanism remains obscure, many studies have shown that the tumorigenesis and progression of bladder cancer are closely related to chromosomal anomalies, epigenetic changes, and genetic polymorphism [3-5], and genetic changes are obviously involved in its initiation and prognosis [6]. Therefore, there is an urgent need to find an effective method to predict prognosis and guide clinical treatments of BLCA.

The tumor microenvironment, which is associated with tumor progression and metastasis $[7,8]$, is comprised of tumor cells and surroundings such as blood vessels, the extracellular matrix, and other nonmalignant cells such as tumor-associated macrophages (TAMs), mesenchymal stem/stromal cells, fibroblasts, pericytes, and immune cells [9]. Among those nonmalignant cells, stromal cells and immune cells play an important role in the whole process of tumors from happening to transferring and have definite clinical significance for diagnosis and prognosis of tumors. In the previous studies, an algorithm called ESTIMATE designed by Yoshihara et al. was used to determine the expression of certain genes of stromal cells and immune cells and calculate immune and stromal scores to infer the fraction of stromal and immune cells in tumor samples and predict the infiltration of nontumor cells $[10,11]$. The previous studies have shown that the ESTIMATE algorithm based on big data is demonstrated effective in numerous cancer tissues, such as prostate cancer [12], breast cancer 
[13], colon cancer [14], and glioblastoma [11]. Though widely applied in varieties of cancer, prognostic evaluation of the ESTIMATE algorithm on BLCA has not yet been completely clarified. Therefore, it provides new opportunities to identify gene expression profile associated with BLCA prognosis. In our study, we took advantage of BLCA cohorts downloaded from TCGA database and ESTIMATE algorithm-derived stromal and immune scores to predict the prognosis of BLCA by a list of microenvironment-associated genes. Subsequently, another cohort of BLCA from ArrayExpress proved the prognostic value of those genes. To further elucidate related immunological mechanisms, we explored the role of the immune microenvironment in the development and prognosis of BLCA by immune cell infiltration analysis.

\section{Materials and Methods}

2.1. Data Source and Preprocessing. In this study, gene expression profiles of and clinical information of 412 patients with bladder cancer were acquired from the TCGA data portal (https://tcga-data.nci.nih.gov/tcga/). The ESTIMATE algorithm was used to calculate the stromal and immune scores and divided the sample patients into two high and low groups separately in accordance with the scores. In order to validate genes with prognostic significance, we downloaded a data set named E-GEOD-13507 containing microarray gene expression data associated with disease prognosis of bladder cancer from ArrayExpress (https://www.ebi.ac.uk/ arrayexpress/). Tumor Immune Estimation Resource (TIMER) (https://cistrome.shinyapps.io/timer/) was used to analyze the correlation between DEGs expression and immune cell infiltration level.

\subsection{Identification of Differentially Expressed Genes (DEGs).} According to the optimal cutoff value of immune/stromal scores through X-title software [15], we divided the patients into low and high score groups. The DEGs between low and high score groups were analyzed with package edgeR [16] in $\mathrm{R}$ language (version 3.5.3). The adjusted $P$ value $<0.05$ and | $\log _{2} \mathrm{FC} \mid>1.5$ were set as the cutoff criteria.

2.3. Heatmaps and Clustering Analysis. The packages ggplot2 and pheatmap were used for the generation of heatmaps [17] and clustering analysis [18].

2.4. Function and Pathway Enrichment Analysis of DEGs. To further explore the biological processes and signal pathways of those DEGs, we performed functional analyses. Gene ontology (GO) gathers information on molecular function (MF), biological processes (BP), and cellular components (CC). Kyoto Encyclopedia of Genes and Genomes (KEGG) pathway analysis was used to excavate remarkable pathways associated with DEGs with prognostic significance. GO and KEGG were performed by R package of clusterProfiler [19]. False discovery rate $(\mathrm{FDR})<0.05$ was considered to be statistically significant.
2.5. Protein-Protein Interactions (PPIs) Network Construction. The DEGs were employed to construct PPIs network by Cytoscape software [20]. The related data were downloaded from STRING database [21] to construct PPI network; then hub genes were obtained. The protein product of DEGs serves as a node in the PPI network, and the interplayed protein numbers reflected the degree of interaction among proteins.

2.6. Survival Curves. The relationship between DEGs and survival was explored by plotting the Kaplan-Meier curve using R. The optimal cutoff value of each DEGs was determined by X-title software. $P<0.05$ was set as the cutoff value for selecting survival-related DEGs.

\section{Result}

3.1. Immune Scores and Stromal Scores Are Remarkably Associated with Smoking, Race, and Bladder Tumor Staging. We obtained biological omics data and clinical information of 412 patients with bladder cancer from TCGA data portal. Among those patients, 108 (26.2\%) patients were female and $304(73.8 \%)$ cases were male. Pathological data included 2 $(0.5 \%)$ cases of stage I, 131 (31.8\%) were stage II, 141 (34.2\%) were stage III, $136(33.0 \%)$ were stage IV, and $2(0.5 \%)$ were not reported. On the basis of ESTIMATE algorithm, immune scores varied from -2023.05 to 3085.28 , and stromal scores ranged from -2628.68 to 2175.37 , respectively. The ESTIMATE algorithm provided us an opportunity to deeply probe into the complex relationship between the immune and stromal scores and the clinical characteristics. The stromal and immune scores showed a rising trend in general along with increasing duration of cigarette smoking (Figures 1(a) and 1(b)). In addition, the White race had the highest averages of immune and stromal scores, followed by that of Blacks and Asians (Figures 1(c) and 1(d)). As the stage evolved, stromal scores increased gradually (Figure 1(f)), indicating that stromal scores are useful indexes to reflect the malignancy of BLCA. However, the scatter plot shows that there is no significant correlation between the degrees of malignancy and immune scores (Figure 1(e)).

According to stromal and immune scores, we divided the BLCA cases into two halves, which are the low levels and the high level, and analyzed potential relationships between scores and survival probability. Kaplan-Meier survival curves (Figure 1(h)) showed that survival probability of cases in the low immune group was lower than the cases in the high score group, although it was not statistically significant. However, lower stromal scores groups showed higher survival probability compared to patients with higher stromal scores (Figure 1(h)).

\subsection{Differentially Expressed Genes Were Obtained through the} Comparison between High and Low Immune/Stromal Score Groups and Used for Further Analysis. By analyzing Affymetrix microarray data of all 412 BLCA cases downloaded from TCGA database, we obtained the differentially 


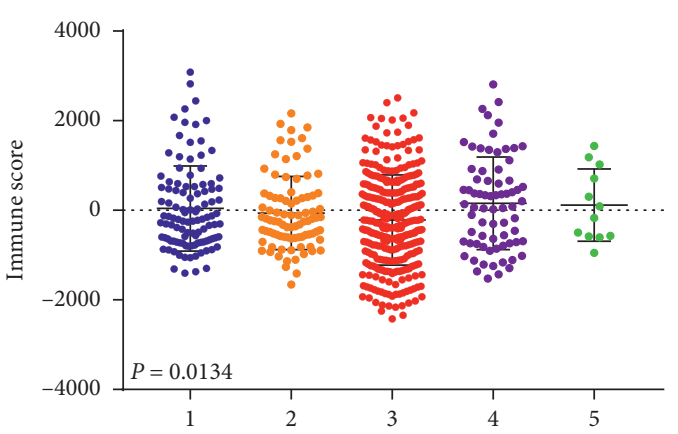

(a)

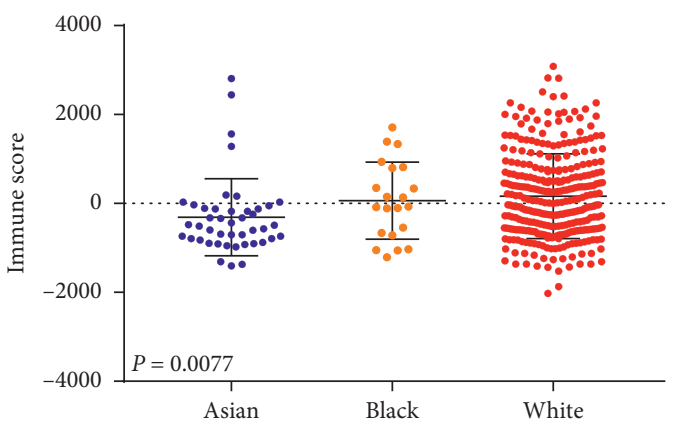

(c)

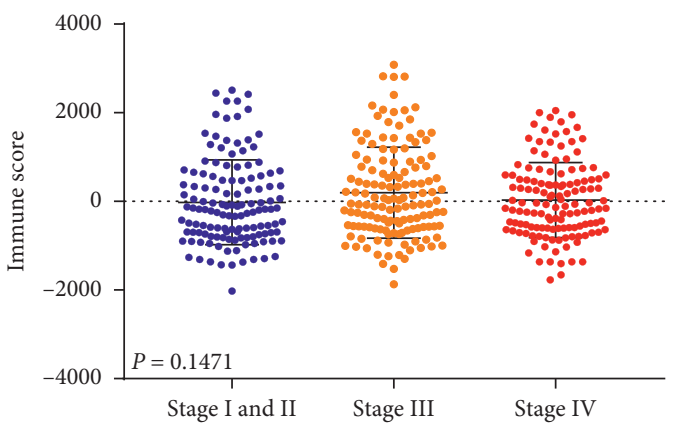

(e)

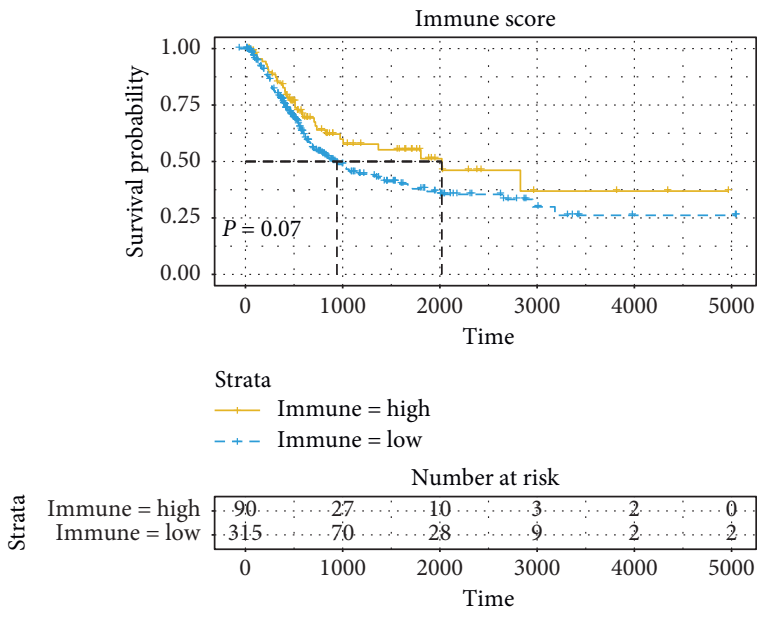

(g)

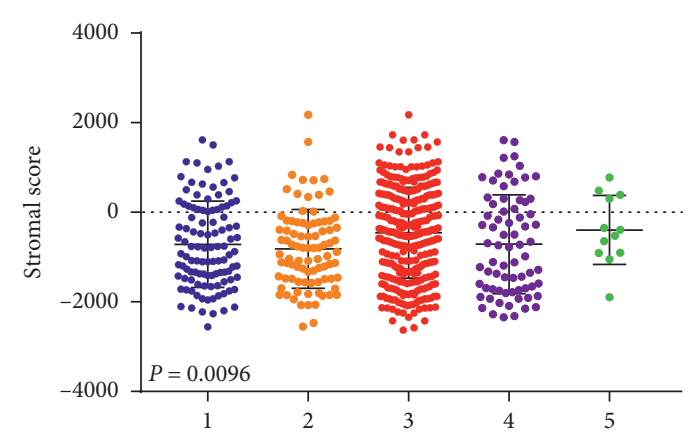

(b)

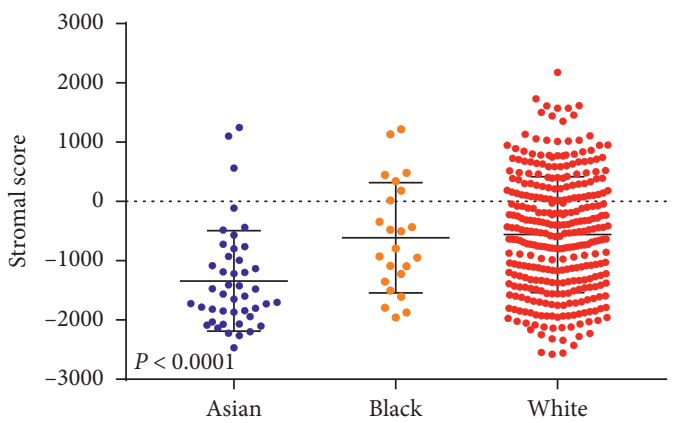

(d)

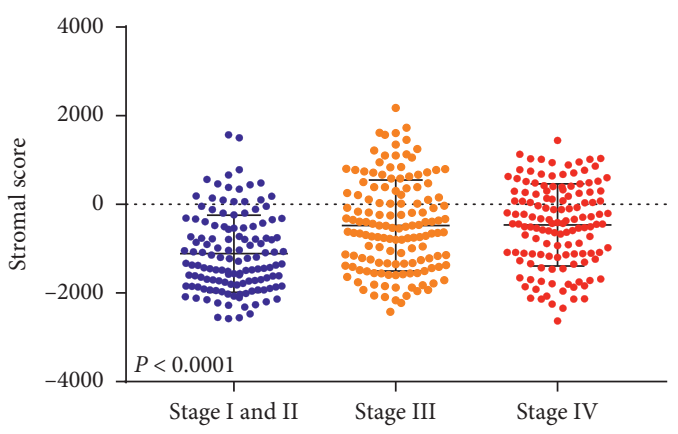

(f)

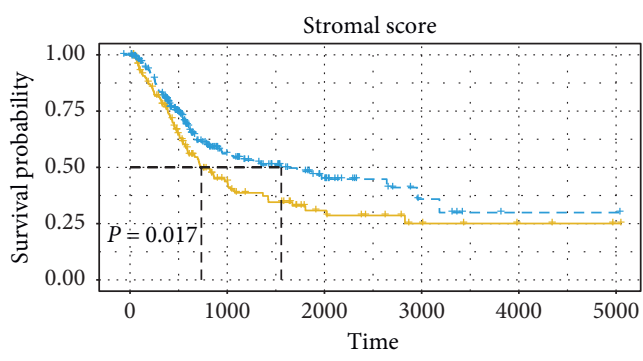

Strata

$\longrightarrow$ Stromal $=$ high

-+- Stromal $=$ low

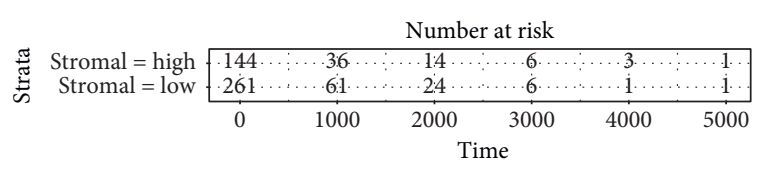

(h)

FIGURE 1: Immune scores and stromal scores are associated with smoking, race, the malignancy, and the survival probability. (A, B) Distribution of immune scores (a) and stromal scores (b) of different duration of cigarette smoking $(n=405, P<0.05)$. (C, D) Distribution of immune scores (c) and stromal scores (d) for Asian, Black, and White race $(n=405, P<0.05)$. (E, F) Distribution of immune scores (e) $(n=405, P=0.1471)$ and stromal scores (f) $(n=405, P<0.05)$ of different degrees of malignancy cases. (G, H) Kaplan-Meier curves for survival probability of bladder cancer patients with low versus high immune scores $(\mathrm{g})(n=405, P=0.07)$ and stromal scores (h) $(n=405$, $P<0.05)$. 
expressed genes (DEGs) through the consolidation and analysis of different gene expression profiles between high and low groups of immune scores and/or stromal scores. Heatmaps showed distinct gene expression profiles of cases, which belonged to high or low immune/stromal scores groups. Genes with higher expression are shown in red and those with lower expression are in green (Figures 2(a) and 2(b)). As the Venn diagrams shows, 483 genes were upregulated, and 1039 genes were downregulated on the basis of stromal scores. Likewise, 834 genes were upregulated, and 849 genes were downregulated on the basis of immune scores. The intersection of stromal and immune group was chosen for further analysis, including 376 coupregulated genes and 492 codownregulated genes (Figures 2(c) and $2(\mathrm{~d}))$.

We further assessed the potential function of the DEGs by functional enrichment analysis of the co-upregulated/ downregulated 868 genes. Molecular function (MF), biological processes (BP), and cellular components (CC) were explored separately based on gene ontology. And the major relevant terms include extracellular matrices, lymphocyte activation, adaptive immune response, leukocyte activation involved in immune response, immunoglobulin binding, and cytokine receptor activity, which provides further evidence that those DEGs were closely related to TME and immune response.

3.3. Excavation of DEGs with Prognostic Value. Based on TCGA database, we constructed survival curve to explore the correlation between DEGs and overall survival. With univariate Cox, survival analysis of 868 DEGs was performed, and 139 of them had statistical significance $(P<0.05)$. Some genes are shown in Figure 3. The expression level of some genes was positively correlated with the overall survival, while some were negative.

3.4. The Value of Those DEGs Related to Prognosis Was Investigated at the Level of Protein. Since protein was the faithful executor of physiological function of the body, we further explored the underlying causal relationships between genes by protein-protein interaction (PPI) networks. The top four remarkable modules (Figure 4) of PPI networks, which were referred to as CD27, TBX21, SLC39A5, and HMHB1 modules for simplicity, were chosen for subsequent assessment. In the CD27 module, the nodes of CD27, PDCD1, GZMA, and SH2D1A had the closest and most extensive contacts with other members of the module. TBX21 module was made up of three immune-related genes, and SLC39A5 module contained several membrane transporter genes. For HMHB1 modules, HMHB1, as one of human minor histocompatibility antigens, had the highest degree values and played a major role in the induction of cytotoxic $\mathrm{T}$ lymphocyte (CTL) reactivity after allotransplantation.

3.5. Functional and Pathway Enrichment Analysis of DEGs Associated with Prognosis. In keeping with PPI network analysis, GO enrichment analysis showed that these DEGs were closely related to stromal elements and immune response. A total of $10 \mathrm{GO}$ terms of cellular component, $20 \mathrm{GO}$ terms of biological process, and 13 GO terms of molecular function were significantly enriched. Important GO terms included apical/basolateral plasma membrane and collagencontaining extracellular matrix (Figure 5(a)), cellular defense response and T cell activation (Figure 5(b)), and BMP binding and collagen binding (Figure 5(c)). Besides, KEGG pathway enrichment analysis was carried, and the result indicated that a set of pathways was enriched and associated with microenvironment and immune response, such as natural killer cell mediated cytotoxicity and cell adhesion molecules (CAMs).

\subsection{Prognostic Value of DEGs Was Verified through Another} BLCA Cohort from ArrayExpress Database. We downloaded and analyzed a data set, named E-GEOD-13507, from ArrayExpress to see if DEGs with prognostic value were applicable for other BLCA cases. The data set was built to study the expression of prognosis-related genes and included 165 primary bladder cancer samples. Finally, a total of 14 genes had proven to be enormously valuable for predicting prognosis of BLCA. Part of the result was shown in Figure 6. The following is all genes: AADACL2, MOGAT2, COMP, KRTAP5-11, FAM57B, DSG1, TNFAIP6, SLC26A5, SPINK4, KRT1, SLC17A1, ATP12A, ERN2, and CTSE.

\subsection{Immune Cell Infiltration Analysis Revealed the Correla-} tions between the Identified DEGs Expression and Immunocyte. To further reveal the role of the immune microenvironment in the development and prognosis of BLCA, we analyzed the correlation between the identified DEGs and immunocyte infiltration. As Figure 7 showed, the expressions of mainly identified DEGs (TNFAIP6, CTSE, COMP, and DSG1) were separately in positive or negative relation to the infiltration level of different immune cells, indicating that the identified DEGs modulated immunologic microenvironment by influencing immune cell infiltration.

\section{Discussion}

Accumulating evidence shows that various components of TME, such as immune cells, soluble factors, and altered extracellular matrix, contribute actively to cancer progression, while linkage between TME-associated genes and cancer prognosis has not been fully elucidated. Analysis of the tumor microenvironment in patients with a variety of solid tumors has revealed that most tumor cells express antigens that can mediate recognition by host CD8+ T cells and must have evaded antitumor immune responses to grow progressively [22]. Additionally, the spontaneous $\mathrm{T}$ cell infiltrate of several solid tumor histologies, including breast cancer [23], renal cell carcinoma, melanoma [24], ovarian cancer, and gastrointestinal stromal tumors [25], may have significant prognostic value. Meanwhile, solid tumor stroma consists of fibroblasts, macrophages, and vascular endothelial cells, with variable amounts of extracellular matrix, all 


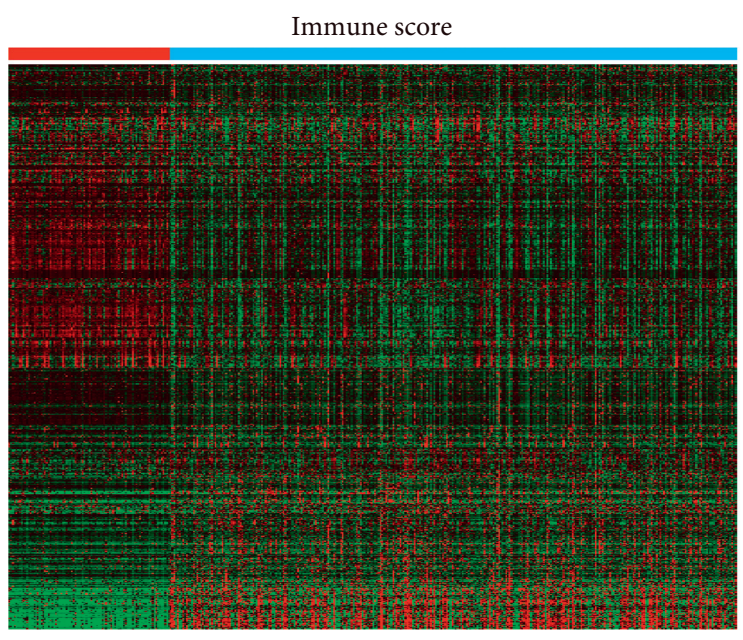

High

Low

(a)

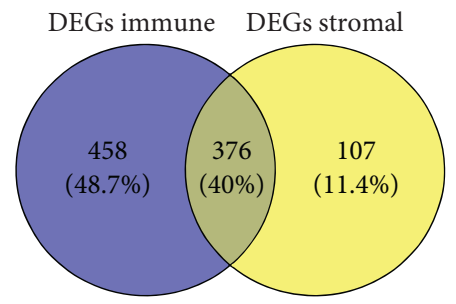

(c)

Cellular component

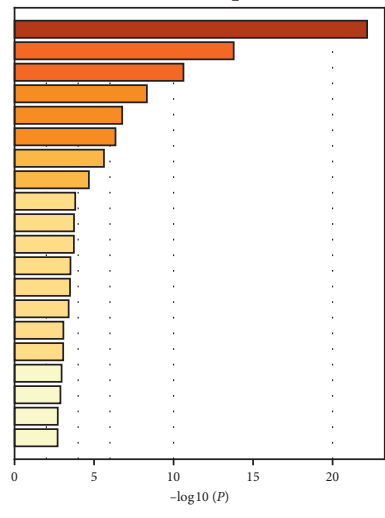

(e)
Stromal score

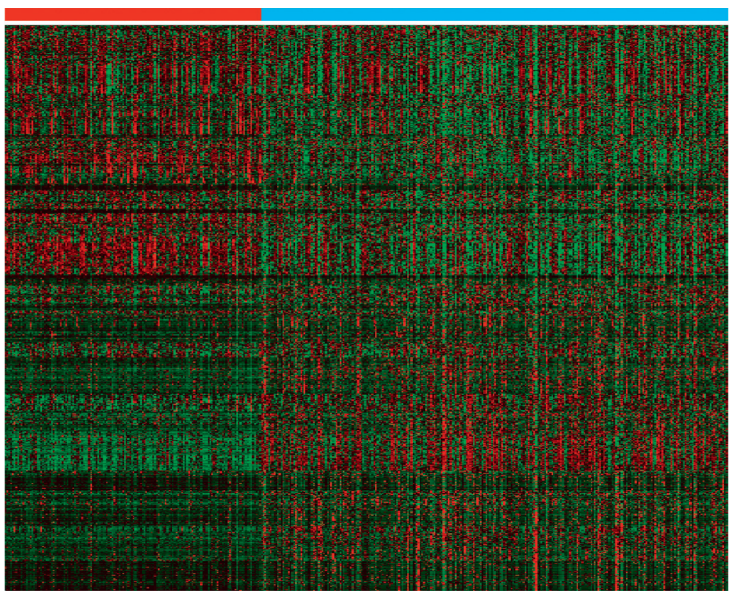

High

Low

(b)

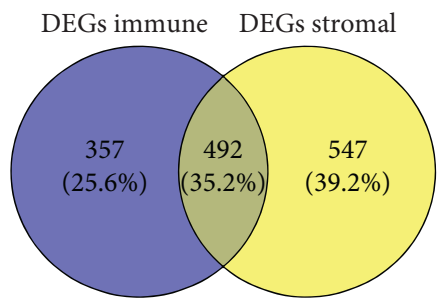

(d)

Biological process

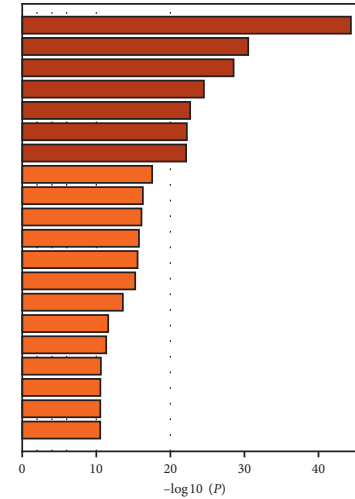

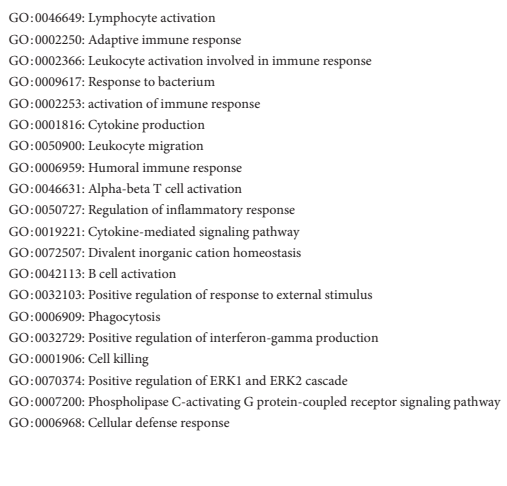

(f)

Figure 2: Continued. 


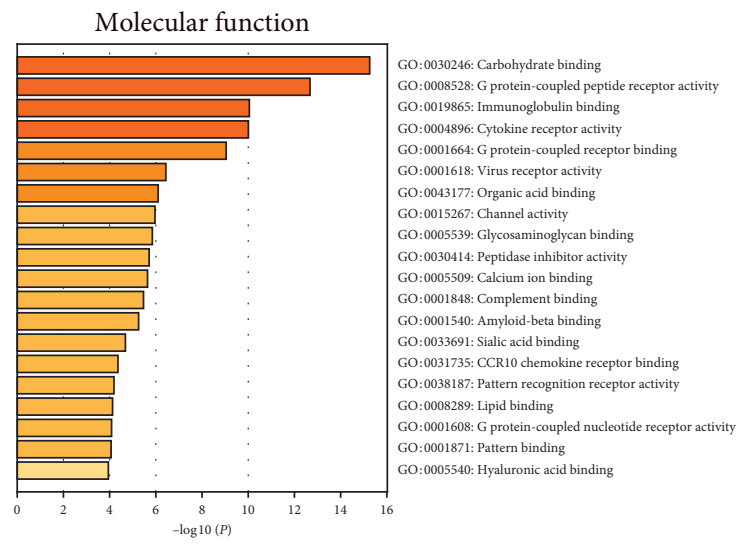

(g)

FIGURE 2: Gene expression profile is of great relevance to immune scores and stromal scores in BLCA. (a, b) Heatmaps show that differentially expressed genes profiles between high and low immune scores/stromal scores groups. Red represents higher expression genes, green represents lower expression genes, black represents same expression genes (fold change $>1.5$ and $P<0.05$ ). (c, d) Venn diagrams show the number of coupregulated (c) or codownregulated (d) DEGs in immune and stromal score groups. (e, $\mathrm{f}$, and g) The major relevant terms. $P<0.05$.

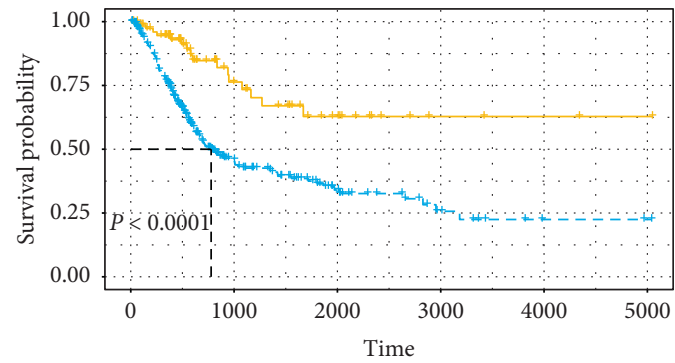

Strata

$\longrightarrow$ CTSE $=$ high

-+ CTSE $=$ low

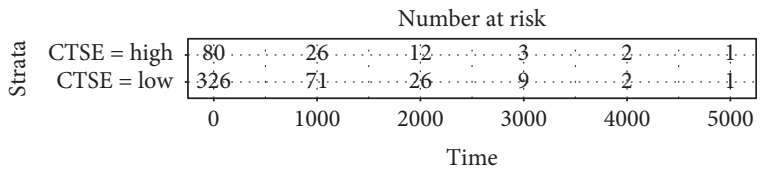

(a)

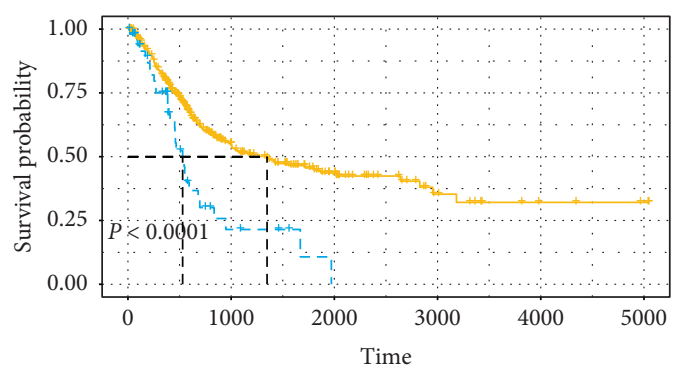

Strata

$\because$ FAM57B = high

- + $\quad$ FAM57B = low

Number at risk

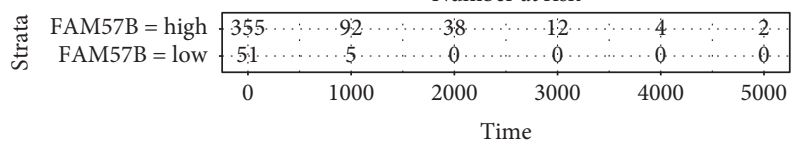

(c)

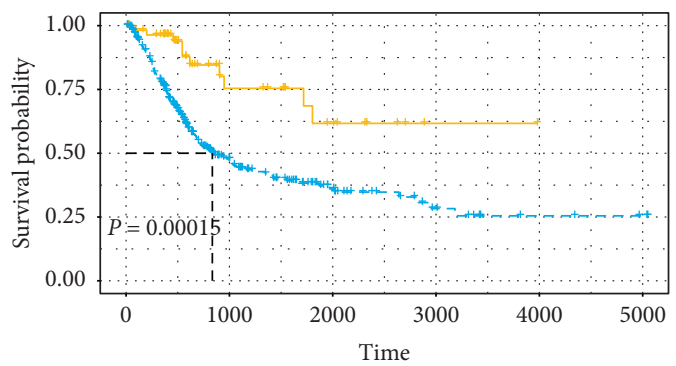

Strata

$\div$ ERN2 = high

- + - ERN2 = low

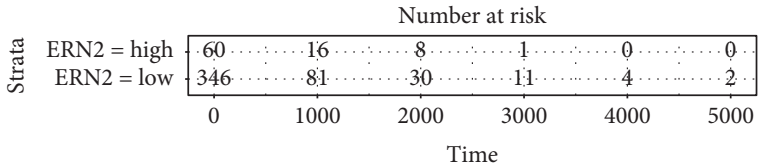

(b)

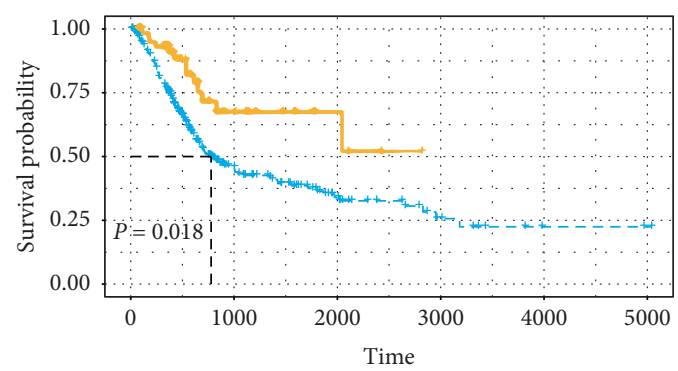

Strata

+ KRTAP5-11 = high

- + - KRTAP5-11 = low

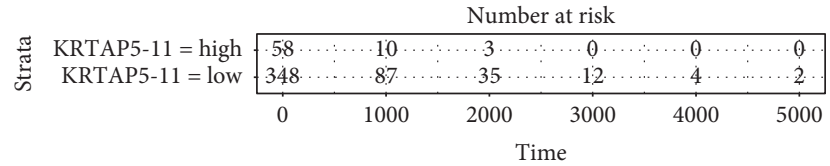

(d)

Figure 3: Continued. 


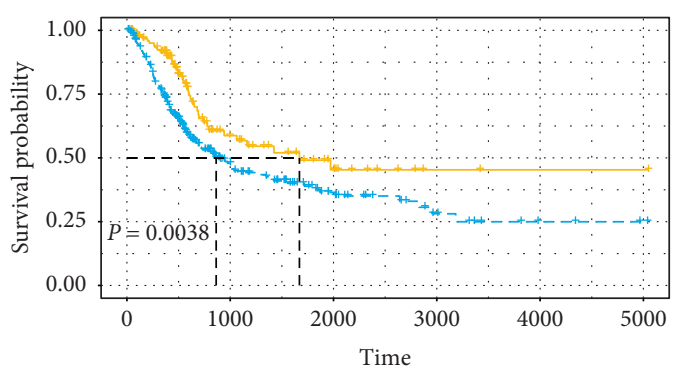

Strata

- MOGAT2 $=$ high

++ MOGAT2 = low

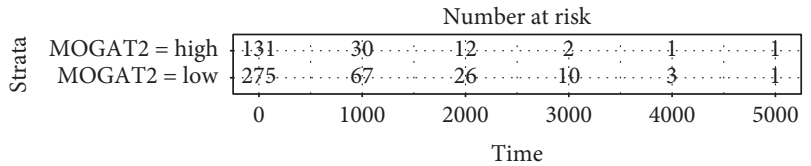

(e)

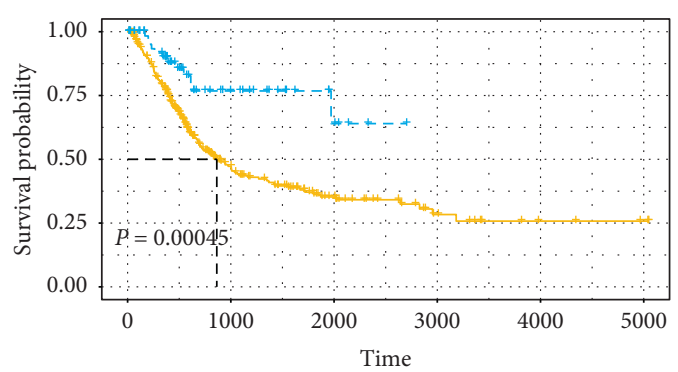

Strata

$+\quad$ TNFAIP6 $=$ high

-+- TNFAIP6 $=$ low

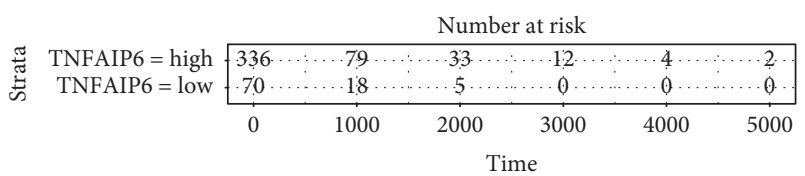

(g)

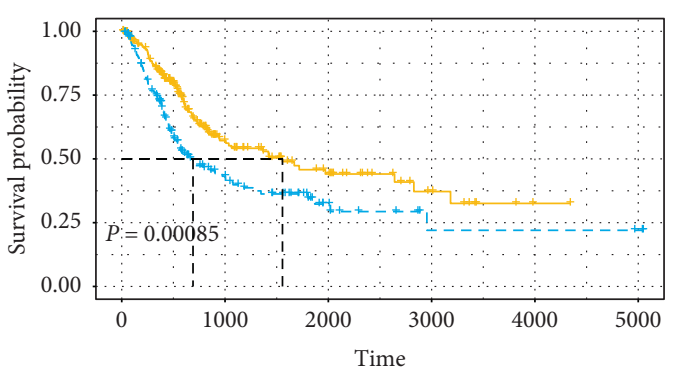

Strata

- SLC26A5 $=$ high

- + SLC26A5 = low

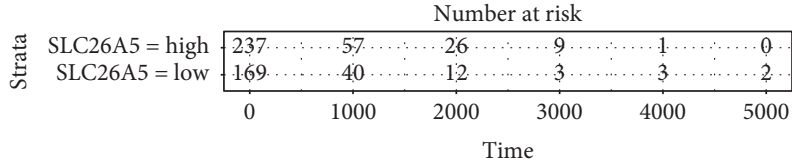

(f)

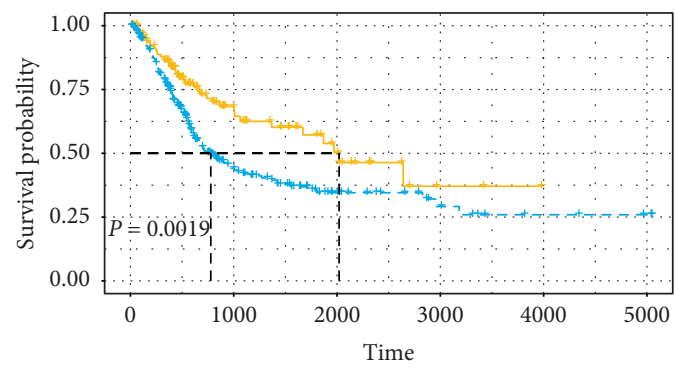

Strata

- SPINK4 $=$ high

- + SPINK4 = low

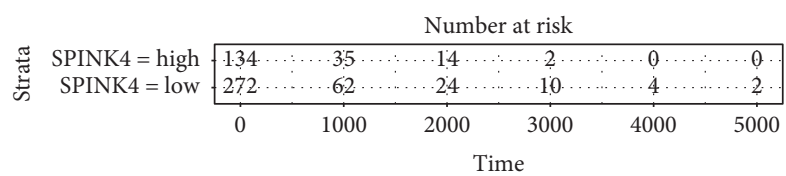

(h)

FIGURE 3: Association between DEGs expressions and overall survival in TCGA. Kaplan-Meier curves for OS $(d)$ of bladder cancer patients with low versus high immune/stromal scores were made to select the DEGs. $P<0.05$.

of which contribute as a support structure for tumor growth. And in addition to significantly regulating tumor growth, these components can impair host immune responses and likely contribute to the degree of immune cell infiltration [26]. Specific to BLCA, although previous findings demonstrated that some immune cells, such as CD3+ tumorinfiltrating lymphocytes (TILs), CD8+ cytotoxic T cells (CTLs), CD68+ TAM [27], and foxp+ regulatory $\mathrm{T}$ cells (Treg) [28], and stromal cells play a vital role in the development of BLCA, few studies have integrated multiple immunological factors into single scores to analyze the significance of immune/stroma-related genes in estimation of the prognosis of BLCA. We downloaded and analyzed microarray gene expression data from TCGA and extracted 139 prognostic DEGs, which were correlated with the stromal element and immune response. To verify whether those DEGs could be applied to other data sets, we took advantage of data from ArrayExpress, a repository to archive functional genomics data from microarray and sequencing platforms, and validated 14 genes with prognostic value in BLCA patients.

Firstly, we analyzed 868 differentially expressed genes extracted from the intersection of stromal and immune score groups. For further understanding of functions involved for the differentially expressed genes, GO analysis was performed and showed that those genes were correlated with stromal element and immune response. Growing evidences have shown that the TME not only influences the ability of growth, invasion, and transfer of tumor cells but also has profound effects on therapeutic efficacy [29]. The current study supported the conclusion that tumor stroma and immune response play a pivotal role in TME-mediated tumor progression.

Afterwards, survival analysis was carried out to demonstrate that 139 of those DEGs had the clinical value of statistically predicting survival probability. Subsequently, we made up 4 modules to comprehend protein-protein interactions and found out that those modules were associated 

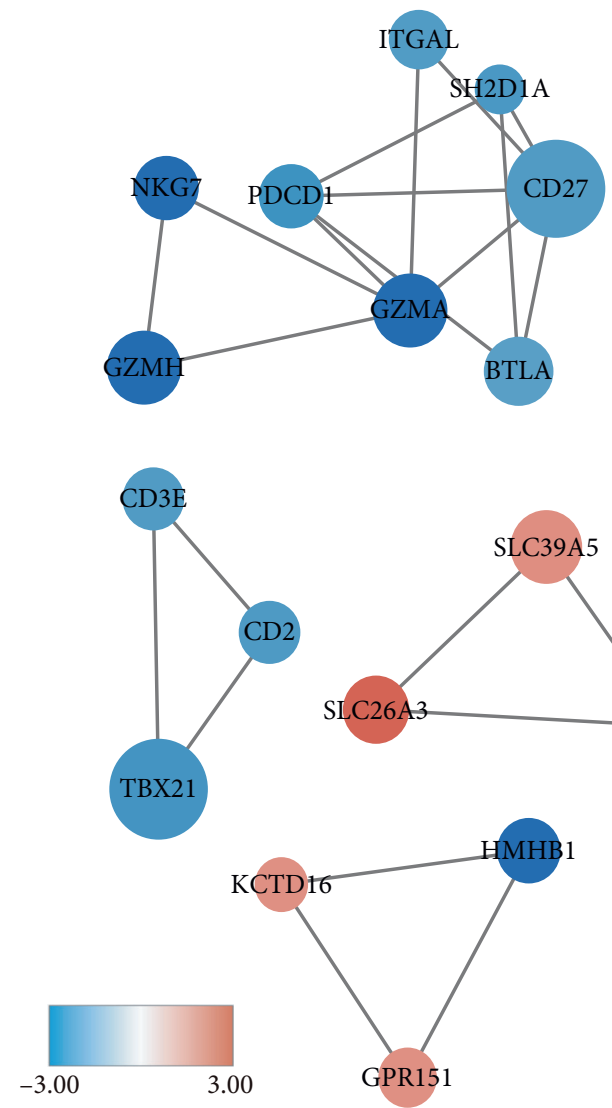

Figure 4: The top four remarkable PPI networks of CD27, TBX21, SLC39A5, and HMHB1 modules.

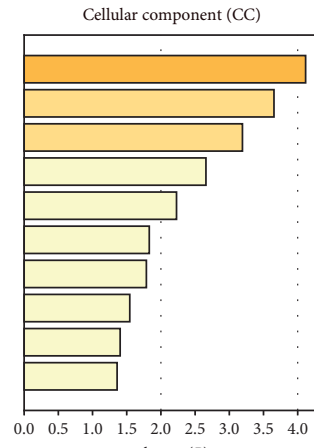

$-\log 10(P)$

Molecular function (MF)

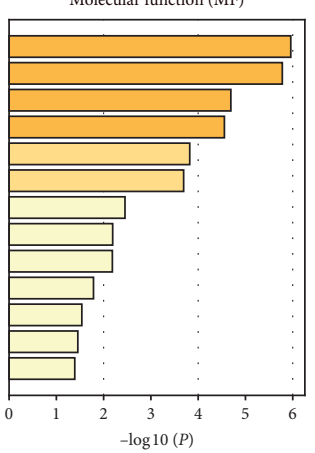

GO:0016324: Apical plasma membrane GO:0016323: Basolateral plasma membrane GO:0101002: Ficolin-1-rich granule GO:0062023: Collagen-containing extracellular matrix GO:0001533: Cornified envelope GO:0031091: Platelet alpha granule GO:0098797: Plasma membrane protein complex GO:0099055: Integral component of postsynaptic membrane GO: 0044297: Cell body GO:0098552: Side of membrane

(a)

GO:0015020: Glucuronosyltransferase activity

GO:0008391: Arachidonic acid monooxygenase activity GO:0019531: Oxalate transmembrane transporter activity GO:0036122: BMP binding

GO:0030246: Carbohydrate binding

GO:0004867: Serine-type endopeptidase inhibitor activity

GO:0046873: Metal ion transmembrane transporter activity GO:0005518: Collagen binding

GO:0005509: Calcium ion binding

GO:0030674: Protein binding, bridging

GO:0031406: Carboxylic acid binding

GO:0005178: Integrin binding

GO:0016747: Transferase activity, transferring acyl groups

other than amino-acyl groups

(c)

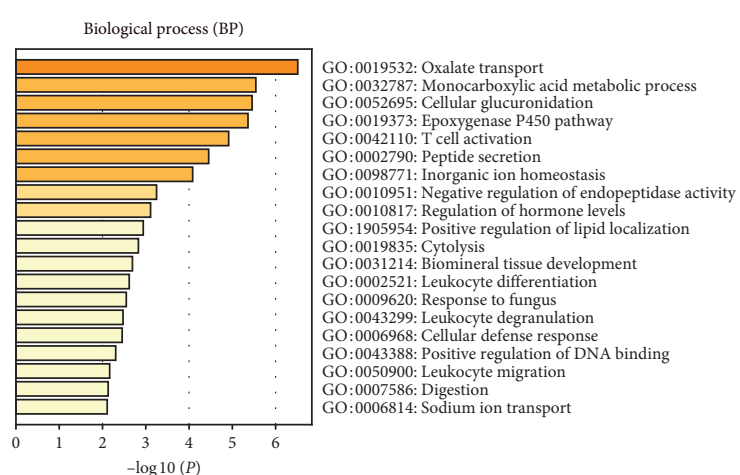

(b)

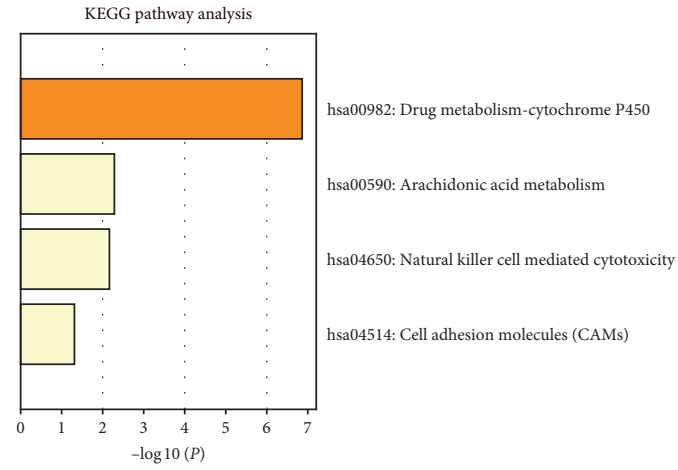

(d)

Figure 5: GO function analysis and KEGG pathway analysis for DEGs associated with overall survival. (a) Cellular components (CC). (b) Biological processes (BP). (c) Molecular functions (MF). (d) KEGG pathways. 


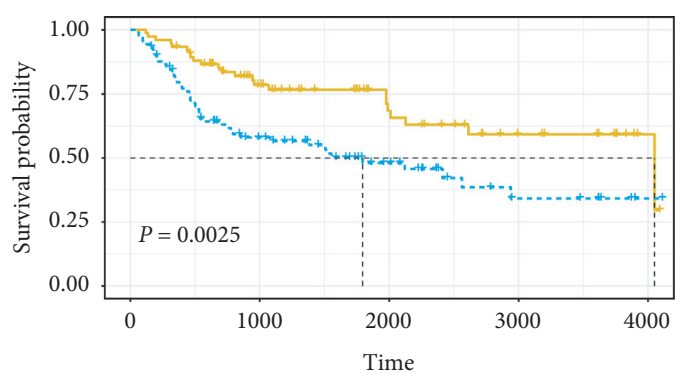

Strata

1- CTSE $=$ high

- + - CTSE $=$ low

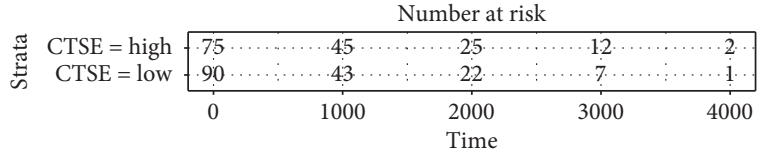

(a)

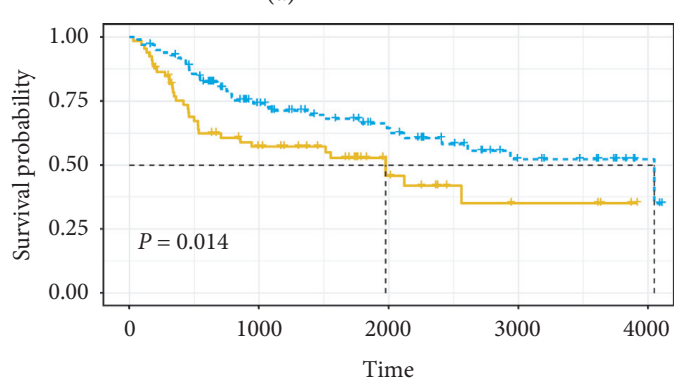

Strata

- FAM57B = high

+ - FAM57B = low

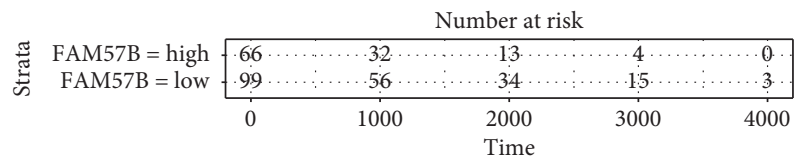

(c)

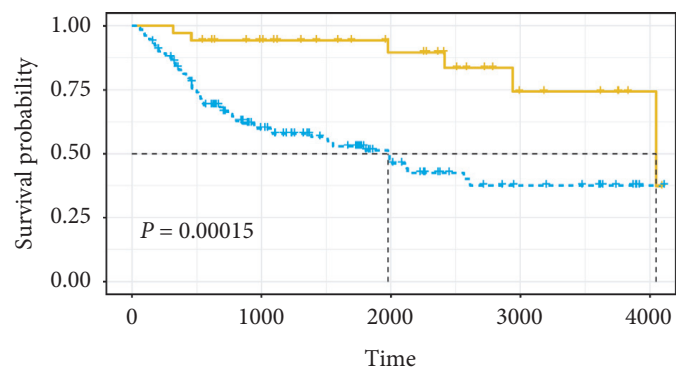

Strata

+ MOGAT2 = high

- + MOGAT2 = low

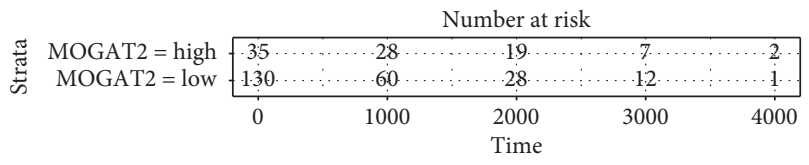

(e)

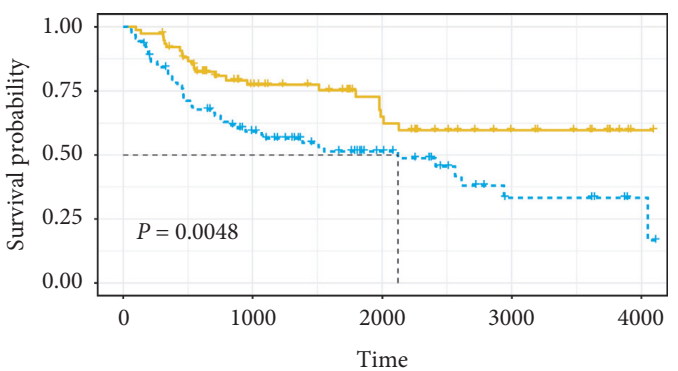

Strata

1- ERN2 = high

- + ERN2 = low

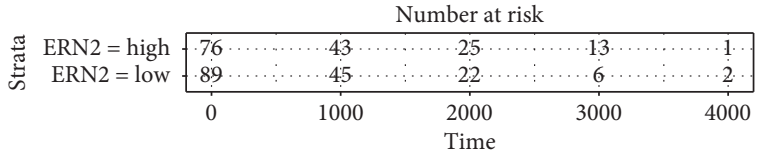

(b)

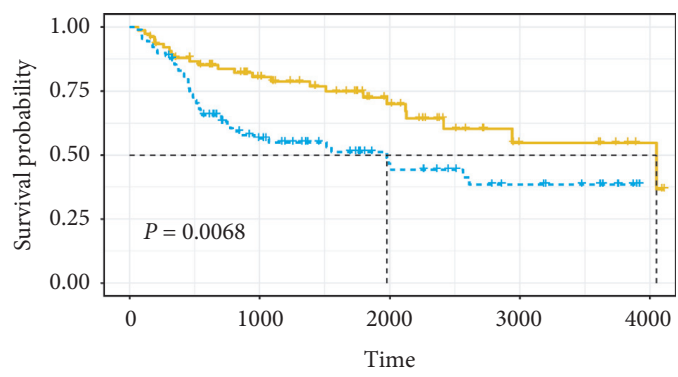

Strata

- KRTAP5-11 = high

- + KRTAP5-11 = low

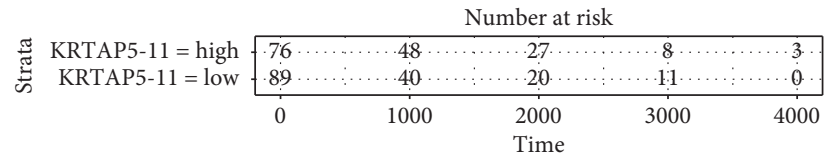

(d)

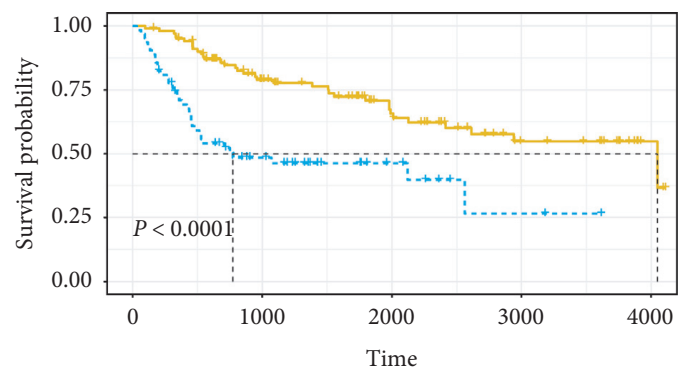

Strata

+ SLC26A5 $=$ high

- + - SLC26A5 = low

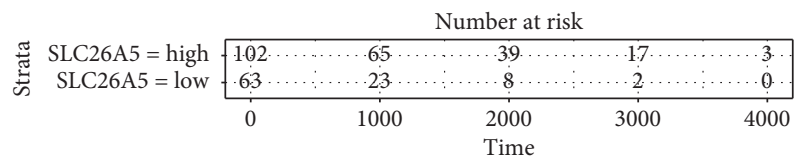

(f)

Figure 6: Continued. 


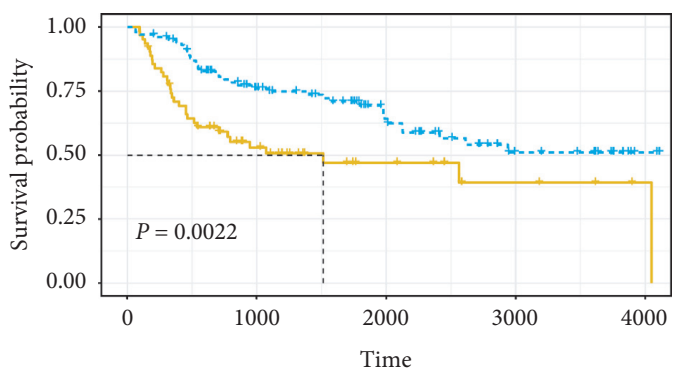

Strata

+ TNFAIP6 $=$ high

- + - TNFAIP6 = low

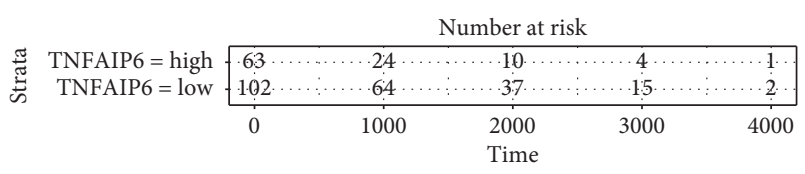

(g)

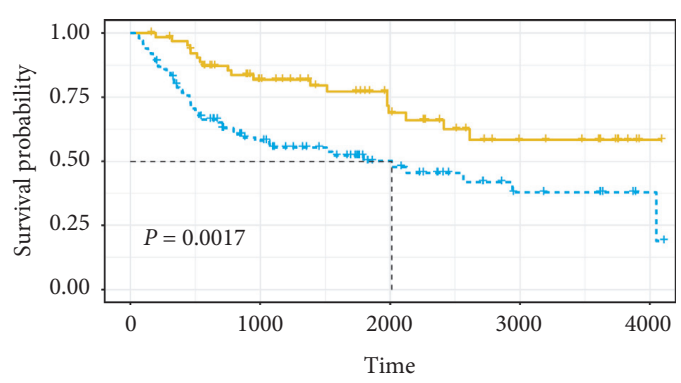

Strata

(1- SPINK4 $=$ high

- + - SPINK4 = low

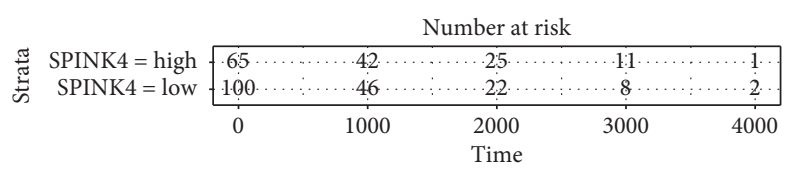

(h)

FIGURE 6: Validation of correlation of DEGs associated with prognosis extracted from TCGA database with overall survival in ArrayExpress database. Kaplan-Meier survival curves of the extracted genes from TCGA were generated to verify the prognostic significance in ArrayExpress database. $P<0.05$.
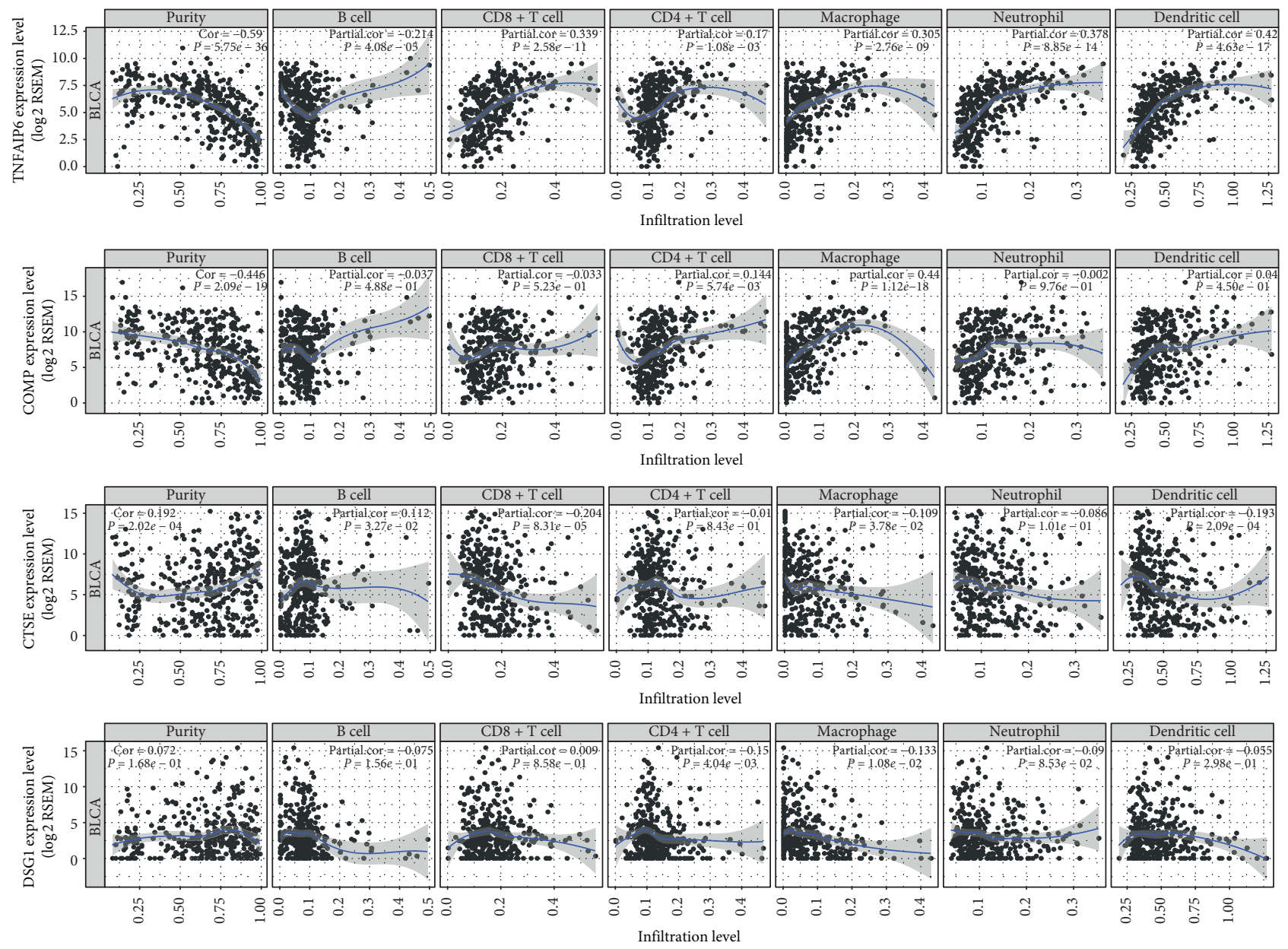

FIGURE 7: Correlation analysis between the expressions of mainly identified DEGs (TNFAIP6, CTSE, COMP, and DSG1) and infiltration levels of B cell, CD8+ T cell, CD4+ T cell, macrophage, neutrophil, and dendritic cell. 
with immune response or transporter. Furthermore, it has been confirmed that the nodes with higher degree values, such as CD27, TBX21, and HMHB1, have a stronger effect on immune cells proliferation and production and induction of cytokines [30-33]. Besides, the SLC39A5, encoding a zinc transporter, is crucial to the maintenance of TME homeostasis because zinc is a critical component of many enzymes involved in hypoxia, angiogenesis, cell proliferation, and cancer metastasis [34]. PPI analysis further strengthens the evidence linking DEGs with prognosis.

To prove the effectiveness of prognostic significance of those DEGs, we analyzed a data set from ArrayExpress, containing gene expression data of 165 primary bladder cancer samples, to see if prognostic significance of the identified DEGs is applicable for other BLCA cases and identified that 14 genes were associated with BLCA prognosis. Besides, studies in the past found that the expression of two genes (TNFAIP6 and CTSE) among those was significantly related to pathological features and strongly associated with overall survival $[35,36]$, suggesting that the selected genes from TCGA and ArrayExpress database based on the algorithm have important clinical value in the promotion of estimating prognosis of bladder cancer. But until now, no study has shown that the remaining 12 genes have notable correlation with BLCA prognosis and could act as a potential prognostic biomarker and therapeutic target in BLCA.

We have paid particular attention to COMP and DSG1 among the remaining genes. As a crucial component of ECM, COMP has the capacity to regulate activation of the complement system and thus innate immunity [37]. In addition, high expression of COMP protects the cells from endoplasmic reticulum (ER) stress, and cells overexpressing the COMP gene undergo a metabolic switch known as the Warburg effect [38, 39]. Furthermore, high COMP expression plays a crucial role in regulating cellular metabolism by blocking intracellular $\mathrm{Ca}^{2+}$ signaling and thus blocking the apoptosis process of the cells [39]. Previous studies have shown that COMP expression in breast cancer cells is significantly associated with poor prognosis [40]. Moreover, COMP expression was also found in colorectal, gastric, lung, ovarian, and pancreatic cancers as shown in the analysis of expression data using the Oncomine database [38]. DSG1, a member of the desmoglein protein subfamily [41], plays a crucial role in cell adhesion [42], whose dysfunction promotes the process of epithelial-mesenchymal transition (EMT) and thus the invasion and metastasis of cancer cells $[43,44]$. It has been shown that, in a variety of tumors such as skin, head and neck, gastric, colorectal, bladder, breast, prostate, cervical, and endometrial cancers, desmosomal proteins were downregulated or even lost, which was associated with poor clinical outcome [45].

One of the basic challenges in cancer is to detect the regulators of gene expression changes in tumorigenesis and the correlation between that and prognosis. The different intracellular signal transduction pathways of low-grade and high-grade tumor heterogeneity in tumor progression and postoperative recurrence are some of the unique characteristics of bladder cancer that contribute to the challenges of evaluating the tumor prognosis [46, 47]. The Cancer Genome Atlas (TCGA) and other large-scale collaborative initiatives collect the comprehensive molecular characterization of multiple cancer types and patients' clinical data and thus provide opportunities for the studies of the genomic and molecular characterization of BLCA $[48,49]$.

The TME plays a vital role in tumor cell proliferation, infiltration, and metastasis and can even determine the extent of malignancy of bladder cancer $[50,51]$. Therefore, the TME components determined by gene expression profiles are one of the determining factors in tumor prognosis. In this study, we integrated the gene expression data associated with extracellular matrix and immune response and obtained a great deal of DEGs with prognostic significance to improve the prediction of BLCA clinical outcomes and explore the interplay between tumor cells and the microenvironment $[29,52,53]$. Additionally, we analyzed the correlation between mainly identified DEGs (TNFAIP6, CTSE, COMP, and DSG1) and immune cell infiltration, whose result proved that the identified DEGs modulated the immune microenvironment by influencing the infiltration level of various immune cells. However, the immune-related signaling pathways and the precise mechanism of those genes affecting progression and prognosis of BLCA remained unknown. In addition to surgical treatment, adjuvant chemotherapy (ACT) is considered as a first-line regimen for advanced or metastatic urothelial bladder cancer. Previous studies have confirmed that the stromal immunotypes could serve as a practical predictive tool to identify pT3 + pT4 patients who would benefit from ACT and be used as a predictor of upcoming popularity of immunotherapy [54]. Accordingly, we could establish an independent prognostic indicator based on immune and stromal scores to analyze whether advanced or metastatic urothelial bladder cancer patients could benefit from ACT [55]. However, due to our limited level, we have not yet retrieved a data set with a sufficiently large sample size and containing ACT information. This will also be the focus of our work in the future.

\section{Conclusions}

In conclusion, we identified novel TME-related gene biomarkers using ESTIMATE algorithm based on immune and stromal scores for predicting clinical outcomes. Besides, we explored the interaction between TME and tumor prognosis, guiding the clinical individualized treatment. However, further studies should be carried out to investigate the molecular mechanisms of TME-related genes affecting prognosis.

\section{Data Availability}

Genes data used to support the findings of this study are included within the article.

\section{Conflicts of Interest}

The authors declare that they have no conflicts of interest. 


\section{Acknowledgments}

Everybody who made a contribution to the research has been listed as an author. This study was supported by financial grants from the Focused Research and Development Program of Shandong Province (Grant no. 2017GSF18162) and the Focused Research and Development Program of Shandong Province (Grant no. GG201809250241).

\section{References}

[1] C. Berdik, "Unlocking bladder cancer," Nature, vol. 551, no. 7679, pp. S34-S35, 2017.

[2] J. Qiu, X. Wang, X. Meng et al., “Attenuated NER expressions of XPF and XPC associated with smoking are involved in the recurrence of bladder cancer," PLoS One, vol. 9, no. 12, Article ID e115224, 2014.

[3] P. Costa-Pinheiro, D. Montezuma, R. Henrique, and C. Jerónimo, "Diagnostic and prognostic epigenetic biomarkers in cancer," Epigenomics, vol. 7, no. 6, pp. 1003-1015, 2015.

[4] H.-T. Li, C. E. Duymich, D. J. Weisenberger, and G. Liang, "Genetic and epigenetic alterations in bladder cancer," International Neurourology Journal, vol. 20, no. 2, pp. S84-S94, 2016.

[5] X. Zhang and Y. Zhang, "Bladder cancer and genetic mutations," Cell Biochemistry and Biophysics, vol. 73, no. 1, pp. 65-69, 2015.

[6] A. Mahdavinezhad, S. H. Mousavi-Bahar, J. Poorolajal et al., "Evaluation of miR-141, miR-200c, miR-30b expression and clinicopathological features of bladder cancer," International Journal of Molecular and Cellular Medicine, vol. 4, no. 1, pp. 32-39, 2015.

[7] G. J. Koelwyn, D. F. Quail, X. Zhang, R. M. White, and L. W. Jones, "Exercise-dependent regulation of the tumour microenvironment," Nature Reviews Cancer, vol. 17, no. 10, pp. 620-632, 2017.

[8] C. Chen, W. He, J. Huang et al., "LNMAT1 promotes lymphatic metastasis of bladder cancer via CCL2 dependent macrophage recruitment," Nature Communications, vol. 9, no. 1, p. 3826, 2018.

[9] W. Meng, S. Xue, and Y. Chen, "The role of CXCL12 in tumor microenvironment," Gene, vol. 641, pp. 105-110, 2018.

[10] K. Yoshihara, M. Shahmoradgoli, E. Martínez et al., "Inferring tumour purity and stromal and immune cell admixture from expression data," Nature Communications, vol. 4, p. 2612, 2013.

[11] D. Jia, S. Li, D. Li, H. Xue, D. Yang, and Y. Liu, "Mining TCGA database for genes of prognostic value in glioblastoma microenvironment," Aging, vol. 10, no. 4, pp. 592-605, 2018.

[12] N. Shah, P. Wang, J. Wongvipat et al., "Regulation of the glucocorticoid receptor via a BET-dependent enhancer drives antiandrogen resistance in prostate cancer," Elife, vol. 6, 2017.

[13] N. Priedigkeit, R. J. Watters, P. C. Lucas et al., "Exome-capture RNA sequencing of decade-old breast cancers and matched decalcified bone metastases," JCI Insight, vol. 2, no. 17, 2017.

[14] M. H. Alonso, S. Aussó, A. Lopez-Doriga et al., "Comprehensive analysis of copy number aberrations in microsatellite stable colon cancer in view of stromal component," British Journal of Cancer, vol. 117, no. 3, pp. 421-431, 2017.

[15] R. L. Camp, M. Dolled-Filhart, and D. L. Rimm, "X-tile: a new bio-informatics tool for biomarker assessment and outcomebased cut-point optimization," Clinical Cancer Research, vol. 10, no. 21, pp. 7252-7259, 2004.
[16] M. D. Robinson, D. J. McCarthy, and G. K. Smyth, "edgeR: a bioconductor package for differential expression analysis of digital gene expression data," Bioinformatics, vol. 26, no. 1, pp. 139-140, 2010.

[17] Z. Gu, R. Eils, and M. Schlesner, "Complex heatmaps reveal patterns and correlations in multidimensional genomic data," Bioinformatics, vol. 32, no. 18, pp. 2847-2849, 2016.

[18] R. Xu and D. C. Wunsch, "Clustering algorithms in biomedical research: a review," IEEE Reviews in Biomedical Engineering, vol. 3, pp. 120-154, 2010.

[19] G. Yu, L.-G. Wang, Y. Han, and Q.-Y. He, “clusterProfiler: an $\mathrm{R}$ package for comparing biological themes among gene clusters," OMICS: A Journal of Integrative Biology, vol. 16, no. 5, pp. 284-287, 2012.

[20] P. Shannon, A. Markiel, O. Ozier et al., "Cytoscape: a software environment for integrated models of biomolecular interaction networks," Genome Research, vol. 13, no. 11, pp. 2498-2504, 2003.

[21] D. Szklarczyk, A. L. Gable, D. Lyon et al., "STRING v11: protein-protein association networks with increased coverage, supporting functional discovery in genome-wide experimental datasets," Nucleic Acids Research, vol. 47, no. D1, pp. D607-D613, 2019.

[22] V. G. Brichard and D. Lejeune, “GSK's antigen-specific cancer immunotherapy programme: pilot results leading to Phase III clinical development," Vaccine, vol. 25, no. 2, pp. B61-B71, 2007.

[23] B. Kreike, M. van Kouwenhove, H. Horlings et al., "Gene expression profiling and histopathological characterization of triple-negative/basal-like breast carcinomas," Breast Cancer Research, vol. 9, no. 5, p. R65, 2007.

[24] F. Azimi, R. A. Scolyer, P. Rumcheva et al., "Tumorinfiltrating lymphocyte grade is an independent predictor of sentinel lymph node status and survival in patients with cutaneous melanoma," Journal of Clinical Oncology, vol. 30, no. 21, pp. 2678-2683, 2012.

[25] S. Rusakiewicz, M. Semeraro, M. Sarabi et al., "Immune infiltrates are prognostic factors in localized gastrointestinal stromal tumors," Cancer Research, vol. 73, no. 12, pp. 34993510, 2013.

[26] T. F. Gajewski, H. Schreiber, and Y.-X. Fu, "Innate and adaptive immune cells in the tumor microenvironment," Nature Immunology, vol. 14, no. 10, pp. 1014-1022, 2013.

[27] G. Sjödahl, K. Lövgren, M. Lauss et al., "Infiltration of CD3+ and CD68+ cells in bladder cancer is subtype specific and affects the outcome of patients with muscle-invasive tumors11Grant support: the Swedish Cancer Society, the Swedish research council, the Nilsson Cancer foundation, the BioCARE Strategic Cancer Research program, the Lund Medical Faculty, and FoU Landstinget Kronoberg and Södra Regionvårdnämnden," Urologic Oncology: Seminars and Original Investigations, vol. 32, no. 6, pp. 791-797, 2014.

[28] A. S. Baras, C. Drake, J.-J. Liu et al., "The ratio of CD8 to Treg tumor-infiltrating lymphocytes is associated with response to cisplatin-based neoadjuvant chemotherapy in patients with muscle invasive urothelial carcinoma of the bladder," Oncoimmunology, vol. 5, no. 5, Article ID e1134412, 2016.

[29] T. Wu and Y. Dai, "Tumor microenvironment and therapeutic response," Cancer Letters, vol. 387, pp. 61-68, 2017.

[30] S. L. Buchan, A. Rogel, and A. Al-Shamkhani, "The immunobiology of CD27 and OX40 and their potential as targets for cancer immunotherapy," Blood, vol. 131, no. 1, pp. 39-48, 2018.

[31] D. Chen, N. Gerasimčik, A. Camponeschi et al., "CD27 expression and its association with clinical outcome in children 
and adults with pro-B acute lymphoblastic leukemia," Blood Cancer Journal, vol. 7, no. 6, p. e575, 2017.

[32] S. Zhao, W. Shen, J. Yu, and L. Wang, "TBX21 predicts prognosis of patients and drives cancer stem cell maintenance via the TBX21-IL-4 pathway in lung adenocarcinoma," Stem Cell Research \& Therapy, vol. 9, no. 1, p. 89, 2018.

[33] M.-J. Park, H.-B. Choi, and T.-G. Kim, "Improved genotyping of the human minor histocompatibility antigen HB-1 by polymerase chain reaction with sequence-specific primers using a complementary oligonucleotide," Tissue Antigens, vol. 76, no. 6, pp. 482-486, 2010.

[34] J. Jin, Z. Li, J. Liu, Y. Wu, X. Gao, and Y. He, "Knockdown of zinc transporter ZIP5 (SLC39A5) expression significantly inhibits human esophageal cancer progression," Oncology Reports, vol. 34, no. 3, pp. 1431-1439, 2015.

[35] T. C. Chan, C.-F. Li, H.-L. Ke et al., "High TNFAIP6 level is associated with poor prognosis of urothelial carcinomas," Urologic Oncology: Seminars and Original Investigations, vol. 37, no. 4, pp. 293 e11-293 e24, 2019.

[36] P. J. Wild, A. Herr, C. Wissmann et al., "Gene expression profiling of progressive papillary noninvasive carcinomas of the urinary bladder," Clinical Cancer Research, vol. 11, no. 12, pp. 4415-4429, 2005.

[37] K. E. Happonen, T. Saxne, A. Aspberg, M. Mörgelin, D. Heinegård, and A. M. Blom, "Regulation of complement by cartilage oligomeric matrix protein allows for a novel molecular diagnostic principle in rheumatoid arthritis," Arthritis \& Rheumatism, vol. 62, no. 12, pp. 3574-3583, 2010.

[38] A. M. Blom, "The role of complement inhibitors beyond controlling inflammation," Journal of Internal Medicine, vol. 282, no. 2, pp. 116-128, 2017.

[39] E. Englund, G. Canesin, K. S. Papadakos et al., "Cartilage oligomeric matrix protein promotes prostate cancer progression by enhancing invasion and disrupting intracellular calcium homeostasis," Oncotarget, vol. 8, no. 58, pp. 9829898311, 2017.

[40] E. Englund, M. Bartoschek, B. Reitsma et al., "Cartilage oligomeric matrix protein contributes to the development and metastasis of breast cancer," Oncogene, vol. 35, no. 43, pp. 5585-5596, 2016.

[41] D. R. Garrod, A. J. Merritt, and Z. Nie, "Desmosomal cadherins," Current Opinion in Cell Biology, vol. 14, no. 5, pp. $537-545,2002$

[42] M. D. Kottke, E. Delva, and A. P. Kowalczyk, "The desmosome: cell science lessons from human diseases," Journal of Cell Science, vol. 119, no. Pt 5, pp. 797-806, 2006.

[43] S. Valastyan and R. A. Weinberg, "Tumor metastasis: molecular insights and evolving paradigms," Cell, vol. 147, no. 2, pp. 275-292, 2011.

[44] J.-M. Peng, R. Bera, C.-Y. Chiou et al., "Actin cytoskeleton remodeling drives epithelial-mesenchymal transition for hepatoma invasion and metastasis in mice," Hepatology, vol. 67, no. 6, pp. 2226-2243, 2018.

[45] R. L. Dusek and L. D. Attardi, "Desmosomes: new perpetrators in tumour suppression," Nature Reviews Cancer, vol. 11, no. 5, pp. 317-323, 2011.

[46] J. Leal, R. Luengo-Fernandez, R. Sullivan, and J. A. Witjes, "Economic burden of bladder cancer across the European union," European Urology, vol. 69, no. 3, pp. 438-447, 2016.

[47] D. S. Morera, M. S. Hennig, A. Talukder et al., "Hyaluronic acid family in bladder cancer: potential prognostic biomarkers and therapeutic targets," British Journal of Cancer, vol. 117, no. 10, pp. 1507-1517, 2017.
[48] D. S. Chandrashekar, B. Bashel, S. A. H. Balasubramanya et al., "UALCAN: a portal for facilitating tumor subgroup gene expression and survival analyses," Neoplasia, vol. 19, no. 8, pp. 649-658, 2017.

[49] K. Tomczak, P. Czerwinska, and M. Wiznerowicz, "The Cancer Genome Atlas (TCGA): an immeasurable source of knowledge," Współczesna Onkologia, vol. 19, no. 1A, pp. A68-A77, 2015.

[50] P. F. Liu, Y.-W. Cao, S.-D. Zhang et al., "A bladder cancer microenvironment simulation system based on a microfluidic co-culture model," Oncotarget, vol. 6, no. 35, pp. 3769537705, 2015.

[51] P. F. Liu, Y. W. Cao, H. P. Jiang et al., "Heterogeneity research in muscle-invasive bladder cancer based on differential protein expression analysis," Medical Oncology, vol. 31, no. 9, p. $21,2014$.

[52] L. Businaro, A. De Ninno, G. Schiavoni et al., "Cross talk between cancer and immune cells: exploring complex dynamics in a microfluidic environment," Lab Chip, vol. 13, no. 2, pp. 229-239, 2013.

[53] M. De Palma and C. E. Lewis, "Macrophage regulation of tumor responses to anticancer therapies," Cancer Cell, vol. 23, no. 3, pp. 277-286, 2013.

[54] H. Fu, Y. Zhu, Y. Wang et al., "Identification and validation of stromal immunotype predict survival and benefit from adjuvant chemotherapy in patients with muscle-invasive bladder cancer," Clinical Cancer Research, vol. 24, no. 13, pp. 3069-3078, 2018.

[55] M. Shahid, T. G. Choi, M. N. Nguyen et al., "An 8-gene signature for prediction of prognosis and chemoresponse in non-small cell lung cancer," Oncotarget, vol. 7, no. 52, pp. 86561-86572, 2016. 